\title{
MODELO DE CERTIFICACIÓN BASADO EN COMPETENCIAS PARA LA CARRERA DE INGENIERÍA EN SISTEMAS INFORMÁTICOS EN EL SALVADOR
}

\author{
A CERTIFICATION MODEL BASED ON COMPETENCES FOR SYSTEMS ENGINEERING \\ PROGRAM IN EL SALVADOR \\ MODELO DE CERTIFICACÃO BASEADO EM CONCORRÊNCIAS PARA A CARREIRA DE \\ ENGENHARIA EM SISTEMAS INFORMÁTICOS EM EL SALVADOR
}

Carlos Roberto Martínez Martínez ${ }^{1}$

Fecha de Recibido: Agosto 01 de 2016 | Fecha de Aprobado: Septiembre 22 de 2016

\begin{abstract}
Resumen
Existe un constante desarrollo en las industrias internacionales del hardware y software a nivel mundial, algunas de esas empresas poseen interés en invertir en El Salvador. Por otra parte, hay un marcado crecimiento en los requerimientos de información que experimentan las empresas del mismo país. Por tales razones, las instituciones de educación superior que se encargan de formar profesionales en el ramo de la informática, deben permanecer atentas a la realidad de su entorno y verificar continuamente el perfil de graduados que debe formarse, con el fin de que los jóvenes puedan enfrentar tanto con efectividad los retos que les esperan y dar un aporte significativo a su sociedad.

En este marco, se realizó un diagnóstico acerca de los perfiles de cargos que son más comunes, las tecnologías más utilizadas y las competencias que para ellas se exigen en términos de las dimensiones cognoscitivas, procedimentales y actitudinales. Según los hallazgos del estudio, hoy en día no basta el simple "saber" acerca de la informática, sino que se debe "saber aprender" o ser un verdadero autodidacta. Luego, el conocimiento debe volverse práctico al aplicarse para la solución de problemas reales. Adicional a esto, las empresas están redescubriendo la importancia de los valores personales y espirituales, como la honestidad, el respeto a los demás, la laboriosidad, adaptabilidad a los cambios, entre otros. Fruto del diagnóstico realizado a relevantes empresas salvadoreñas, se creó un modelo basado en competencias para la educación superior en tecnologías de la información.
\end{abstract}

Palabras clave: Sistemas, informática, computación, software, hardware, empresas, competencias, programación, bases de datos, educación, ingeniería, redes, universidad.

1 Ingeniero en sistemas informáticos. Docente, Facultad de Ingeniería y Arquitectura. Universidad Católica de El Salvador. Correo electrónico: carlos.martinez@catolica.edu.sv 


\begin{abstract}
Currently, international industries of hardware and software have a continuous development, some of them are interested in investing in El Salvador. On the other hand, there is a remarkable increase of information requests experimented by the companies of this country. For that reason, high schoolinstitutesthat are responsible for educating professionals in computing, must consider the reality of its environment and identify the profile of the graduated in training. Thus, the aim is to prepare young students to face the upcoming challenges with efficacy and contribute to their society.

Within this framework, a diagnosis was made to know the most common positions, the most-used technologies and the competences required in terms of cognitive, procedural and attitudinal dimensions. According to this study findings, nowadays the simple "know" about computing is not enough, it is necessary to "know how to learn" or to be a real self- taught person. Then, the knowledge must become pragmatic to be applied in real problem solving. In addition, companies are rediscovering the significance of personal and spiritual valuessuch as honesty, respect, arduousness, adaptabilityto changes, among others. As product of the diagnosis made to relevant Salvadorean companies, a model based on competences for higher education in information technologies was created.
\end{abstract}

Keywords: Systems, computing, software, hardware, companies, competences, programming, databases, education, engineering, networks, university.

\title{
Resumo
}

Existe um constante desenvolvimento nas indústrias internacionais do hardware e software a nível mundial, algumas dessas empresas possuem interesse em investir em El Salvador. Por outra parte, há um marcado crescimento nos requerimentos de informação que experimentam as empresas do mesmo país. Por tais razões, as instituições de educação superior que se encarregam de formar profissionais no ramo da informática, devem permanecer atenta à realidade de seu meio e verificar continuamente o perfil de graduados que deve ser formado, com o fim de que os jovens possam enfrentar tanto com efetividade os desafios que thes esperam e dar uma contribuição significativa a sua sociedade.

Neste marco, realizou-se um diagnóstico a respeito dos perfis de postos que são mais comuns, as tecnologias mais utilizadas e as concorrências que para elas se exigem em termos das dimensões cognoscitivas, processuais e atitudinal. Segundo os achados do estudo, hoje em dia não basta o simples "saber" a respeito da informática, senão que se deve "saber aprender" ou ser um verdadeiro autodidata. Depois, o conhecimento deve ser voltado prático ao aplicar para a solução de problemas reais. Adicional a isto, as empresas estão re descobrindo a importância dos valores pessoais e espirituais, como a honestidade, ou respeito aos demais, a laboriosidad, adaptabilidade às mudanças, entre outros. Fruto do diagnóstico realizado a relevantes empresas salvadoreñas, criou-se um modelo baseado em concorrências para a educação superior em tecnologias da informação.

Palavras chave: Sistemas, informática, computação, software, hardware, empresas, concorrências, programação, bancos de dados, educação, engenharia, redes, universidade. 


\section{INTRODUCCIÓN}

Es indiscutible que la formación académica es de gran valor. Sin embargo, obtener un título universitario no es sinónimo de poseer todas las habilidades y conocimientos concretos que la sociedad demanda de los profesionales. La Ingeniería en Sistemas Informáticos es aún más susceptible de este fenómeno, debido a las constantes modernizaciones en las industrias del hardware y el software. La creación de un modelo de certificación basado en competencias, a partir de un diagnóstico apropiado, hará posible que los nuevos estudiantes encaucen mejor su formación (Sánchez, 2008; Comisión Económica para América Latina y el Caribe- CEPAL \& Organización Internacional del Trabajo- oIT, 2015), al aclarar lo que en realidad se espera de ellos cuando finalicen sus estudios. Dicho modelo también contribuye con los docentes, para la orientación de sus cátedras y todo tipo de programa de capacitación o certificación en el ramo que la Universidad impulse en el futuro.

Es importante recalcar que al momento de realizar el presente diagnóstico, no se encontraron antecedentes relevantes, ya que la educación universitaria ha venido siguiendo un modelo tradicional que no evalúa competencias, sino conocimientos generales relativos a diversas temáticas (Zalba, Gutierrez \& Deamici, 2010), de manera que gran cantidad de egresados de Ingeniería en Sistemas Informáticos no son capaces de desarrollar software, tal como lo manifestaron las empresas que fueron abordadas durante este estudio.

\section{METODOLOGÍA}

La investigación fue descriptiva. A través de informes oficiales y sondeos de mercado se determinó el tipo y la cantidad de empresas e instituciones a consultar. Luego, se diseñaron y validaron los instrumentos de recopilación de datos acerca de los conocimientos más relevantes que se requerían de los ingenieros en sistemas informáticos.

En el ámbito del muestreo, de acuerdo a la última versión disponible del Directorio de Empresas (2011) y de la Dirección General de Estadísticas y Censos (DIGESTYC) $^{2}$, se contabilizaron aproximadamente 20 mil empresas privadas en el país. Se usó una fórmula matemática para el cálculo del tamaño de la muestra (Bernal 2006):

2 Recuperado de: http://www.digestyc.gob.sv/index.php/noveda des/publicaciones/category/45-directorio-de-empresas.html
Figura 1. Fórmula matemática para el cálculo del tamaño de la muestra

$$
\mathrm{n}=\frac{\mathrm{N} \cdot \mathrm{Z}_{\alpha}^{2} \cdot \mathrm{p} \cdot(1-p)}{\mathrm{e}^{2} \cdot(\mathrm{N}-1)+Z_{\alpha}^{2} \cdot p \cdot(1-p)}
$$

Fuente: Bernal (2006).

Donde,

- $\mathrm{n}=$ tamaño de la muestra.

- $N$ = número de elementos de la población, que es 20 mil.

- $\mathrm{Z}^{2}=$ nivel de confianza, ponderado del 95\%, correspondiendo a un valor de 1.96 .

- $p=$ variable de proporción de elementos de la población con una característica. Se adopta el valor estándar de $50 \%$.

- e = error estadístico o precisión de $\pm 5 \%$

Dicha fórmula sugiere una muestra de 303 empresas.

El instrumento de recopilación de datos fue aplicado al personal coordinador de las áreas de sistemas informáticos, por vía telefónica y medios electrónicos. La información fue vaciada sobre una base de datos de PostgreSQL, especialmente diseñada para el estudio. No fue necesario realizar visitas personalizadas a empresas, ya que este tipo de profesionales está acostumbrado a comunicarse remotamente, y una visita presencial se considera como un evento que consume mayor tiempo.

Obtenidos los datos, se procedió a determinar las habilidades y competencias específicas para los diversos roles, en los cuales se espera que se desenvuelvan los profesionales del ramo, así como también se comparó los resultados con estudios realizados anteriormente en otros países (Juan, A, 2006; Villa, 2007; Giraldo, 2009; Simón, 2010; Cataldi, 2007). Tales competencias fueron agrupadas de acuerdo a las más comunes especializaciones de la informática.

\section{RESULTADOS}

\section{Empresas sondeadas}

Durante el estudio, se abordaron 304 empresas que se tipifican como medianas a grandes. No se tomaron en cuenta pequeñas o micro empresas que 
no hacen uso de tecnología informática, ya que por tal razón no requieren de profesionales en el área. Es relevante aclarar que la mayor cantidad de las empresas que usan tecnología, son del tipo "servicios", que representa el $32.8 \%$, en contraste con las empresas del sector de producción que conforma un $10.8 \%$, lo cual evidencia la ya conocida problemática salvadoreña acerca de la necesidad de incrementar la inversión en materia de producción, con el fin de reactivar la economía.

La muestra seleccionada comprendió un 3.6\% de empresas de capital extranjero que se sitúan en nuestro país como transnacionales. Otro $5.6 \%$ lo constituyen instituciones financieras como bancos, cajas de crédito y semejantes. El $19 \%$ de las empresas de gran comercio se formó por entidades que obtienen beneficios económicos de la importación y reventa de productos terminados. Finalmente, las empresas que se dedican a ofertar recurso humano tecnificado, son aquellas firmas de consultoría que usan su fuerza laboral para brindar soluciones informáticas a terceros o que prestan servicios bajo la modalidad conocida como "outsourcing". En la rama de la informática, dichas empresas tienen protagonismo en El Salvador y fueron el $24.6 \%$ de la muestra. Un sub segmento de éstas operan como agencias de reclutamiento. La figura 2 resume estos resultados:

Figura 2. Tipos de instituciones que constituyeron la muestra del estudio

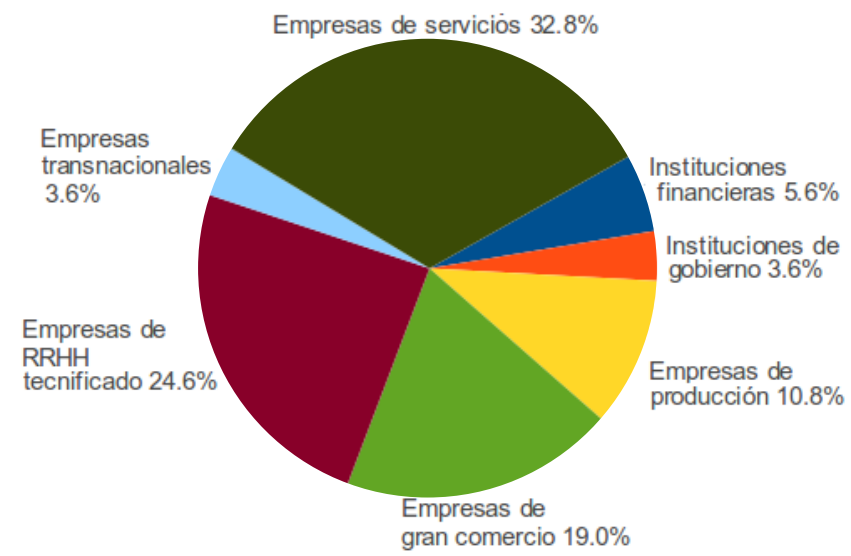

Fuente: elaboración propia.

\section{Sistemas operativos de servidor}

Los sistemas operativos de servidor más utilizados son de tipo "propietario". Éstos abarcaron el 63\% de la muestra y entre ellos se puede mencionar a
Microsoft Windows Server como líder, OS/400 de IBM, AIX de IBM, Solaris de Oracle Corporation y Unix. Un rival importante para estos sistemas de código cerrado, es GNU/Linux, basado en software libre, usado en un $37 \%$ de las empresas abordadas (Ver figura 3).

No se hizo mayor distinción entre las numerosas distribuciones de GNU/Linux, excepto que las más usadas son Oracle Linux, RedHat, Debian y Ubuntu, debido a que el objetivo central de este estudio fue determinar competencias técnicas, y puesto que todas ellas tienen grandes semejanzas entre sí, quien sea conocedor de alguna, puede adaptarse con relativa facilidad a las demás.

Para aprovechar distribuciones como Debian y Ubuntu, no se requiere de algún pago, y, además, suelen tener muy buen rendimiento y robustas características de seguridad; sin embargo, las empresas de El Salvador optan por pagar caras licencias de software propietario por diversas razones, tales como la costumbre, las estrategias de mercado de los representantes de marca, la cantidad de informáticos capacitados en sistemas propietarios y la oferta de soporte técnico por parte del fabricante, aun cuando en la marcha, este último es usualmente brindado a cambio de cargos económicos adicionales, relativamente altos comparados con el costo original de la licencia.

Figura 3. Sistemas operativos de tipo "Servidor", que son usados por las empresas

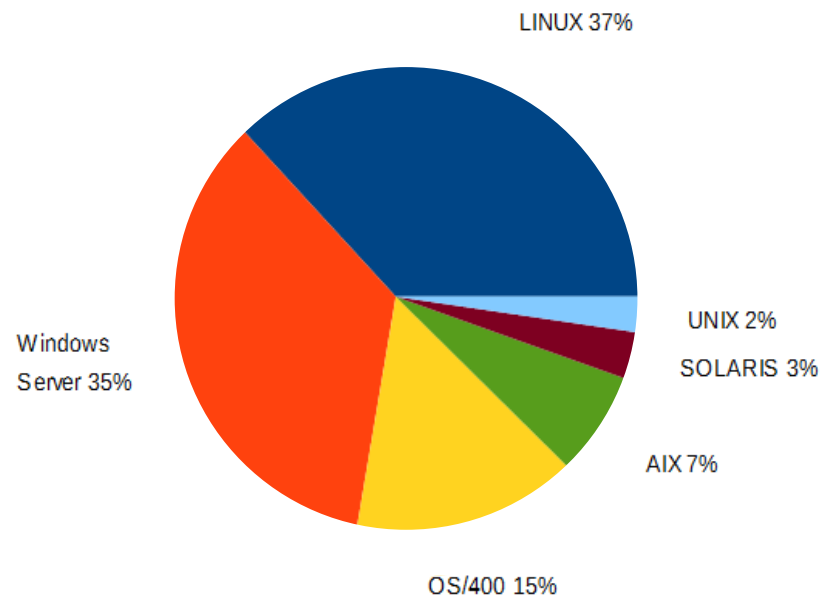

Fuente: elaboración propia.

\section{Tecnologías móviles}

Una de las tecnologías que ha tenido un avance a nivel mundial es la de aplicaciones para dispositivos 
móviles que se ejecutan en Android e iOS; no obstante, a pesar de su avance en otros países, en El Salvador aún se manifiesta como una tendencia emergente. Sólo un $8.5 \%$ de las empresas requieren personal especializado para programación de móviles, siendo Android el dominante, debido al precio bajo de sus aparatos. Empero, este pequeño porcentaje ha surgido en pocos años y debido a las tendencias internacionales, se espera que experimente un rápido crecimiento. Como ejemplo puede mencionarse que varias empresas reconocidas ya ofrecen sus productos comerciales o servicios a través de aplicaciones para Android. Incluso, instituciones del Estado han puesto a disposición gratuita ciertas aplicaciones. Se determinó que el 6\% de las empresas ya se encuentran desarrollando aplicaciones para Android, preferiblemente a través de Android Studio. En la figura 4 puede apreciarse el avance de las tecnologías "mobile" o móviles en El Salvador.

Figura 4. Sistemas operativos móviles que son usados como plataforma de desarrollo de aplicaciones por las empresas salvadoreñas

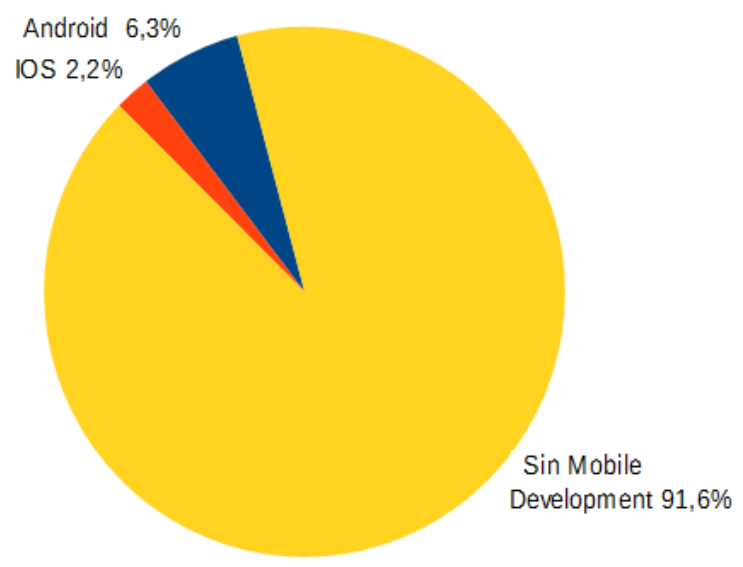

Fuente: elaboración propia.

\section{Lenguajes de programación}

En cuanto a los lenguajes de programación que usan las empresas (ver figura 5), el de mayor expansión es Java. El $40 \%$ de la muestra utiliza éste o alguno de sus componentes derivados, mientras que un $25 \%$ usa productos de su rival más cercano que es Microsoft.NET.

Cuando se recolectaron datos de empresas consultoras $^{3}$ y agencias de outsourcing, se observó que el

\footnotetext{
3 Dedicadas sobre todo a maquilar software para terceros.
}

requerimiento de personal competente en Java era del doble o hasta el triple en comparación con otros lenguajes de programación, es decir, que dicha tecnología no sólo es usada en una mayor cantidad de empresas, sino que también es la que ofrece mayor cantidad de puestos laborales en cada una de ellas.

En el área de Enterprise Resource Planning (ERP) se pudo observar que SAP ha tenido un avance limitado en cuanto a la cantidad de empresas que lo usan, sólo el $6 \%$, debido al costo oneroso de su licencia; ahora bien, es necesario aclarar que actualmente ésta se encuentra funcionando en algunas de las firmas más grandes del país, por lo cual puede decirse que su presencia es significativa en el mercado nacional. El lenguaje predeterminado para programar en SAP es Advanced Business Application Programming (ABAP); sin embargo, éste no está ampliamente documentado en Internet y usualmente los programadores deben ser capacitados por terceros antes de empezar a trabajar, lo cual implica desembolsos económicos adicionales para la empresa.

Otro lenguaje de programación que es muy usado en el extranjero es Objective C, para Mac os X, IOS y Gnustep. Según la muestra obtenida, sólo un $1 \%$ de las empresas está usando este lenguaje, pero se considera que éste valor tenderá a la alza mientras se mantenga la popularidad de los dispositivos que corren el sistema operativo Ios.

Figura 5. Lenguajes de programación usados por las empresas de El Salvador

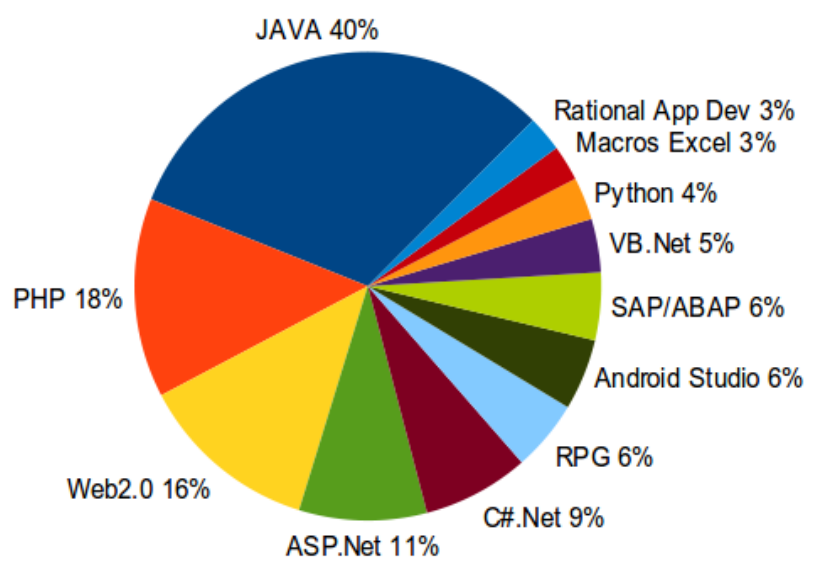

Fuente: elaboración propia.

Report Program Generator (RGP), es un caso similar al de SAP/ABAP. Sólo el 6\% de las empresas abordadas manifestaron utilizarlo, pero tales entidades tienen 
larga trayectoria y alcance a nivel nacional, por lo cual su presencia aún puede considerarse relevante.

El lenguaje PHP ha logrado mantenerse en el mercado con un $18 \%$ de participación por ser una plataforma de uso libre para el desarrollo Web, dotada de un rendimiento excelente. Ésta se usa en empresas comerciales, instituciones educativas y oNG para informar a los usuarios de Internet acerca de sus servicios. Algunas empresas lo han utilizado para crear portales de comercio electrónico. Diverge en cambio, el caso de la banca, profesionales entrevistados manifestaron que se ha optado por utilizar Microsoft ASP.Net bajo el argumento que éste facilita la gestión de la seguridad y la escalabilidad ${ }^{4}$, aunque su rapidez en procesamiento de datos sea inferior.

La figura 6 indica que el 16\% de las empresas utilizan tecnologías para el desarrollo de sitios que cumplen con los estándares Web 2.0. De dicho segmento de la muestra, las tecnologías más usadas son Javascript y HTML5, en el $100 \%$ de los casos. El $94 \%$ usa JQuery, el $90 \%$ usa css y el $75 \%$ usa WebServices basados en el protocolo Simple Object Access Protocol, SOAP.

Figura 6. Uso de tecnologías de desarrollo Web 2.0

4 Tal teoría fue objetada por los expertos en PHP que participaron del estudio.
Microsoft ASP. Net MVc, con licencia propietaria, y que abarca sólo un tercio de empresas comparado con sus dos competidores ya mencionados.

Figura 7. Uso de frameworks de desarrollo

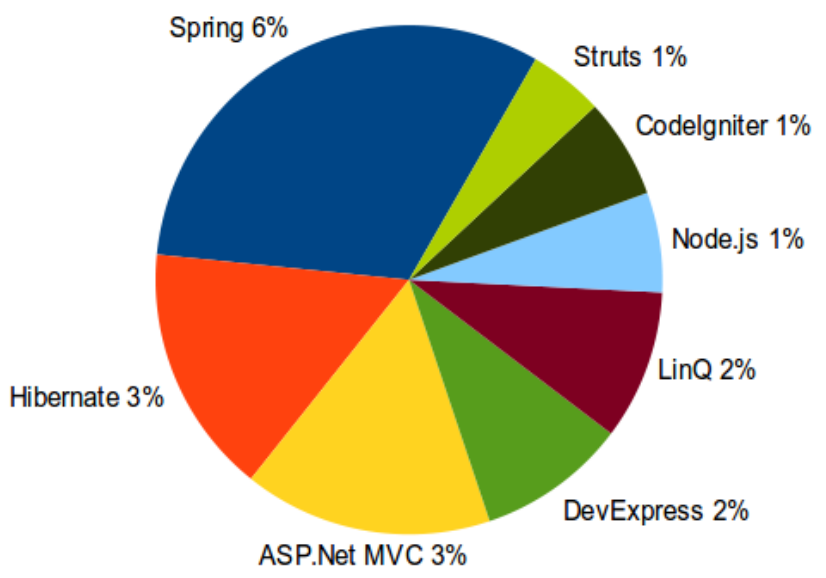

Fuente: elaboración propia.

\section{Servidores de bases de datos}

En cuanto a gestores de bases de datos (ver figura 8 ), Oracle se encuentra presente en la mayoría de empresas. Su porcentaje es del $39 \%$. A esto se le suma el hecho que no sólo se utilizan los servicios propios del motor SQL, sino también otros complementos como las herramientas para crear formularios, reportes, copias de respaldo, funciones para clúster, entre otros. Microsoft SQL Server va en segundo lugar con un $32 \%$; también integra todos esos servicios a un menor costo, aunque su rendimiento es considerado por algunos como inferior al de Oracle. M YSQUL, también propiedad de Oracle Corporation, es usado en un $18 \%$ debido a su alta velocidad de operación y que puede ser utilizado a cualquier escala sin la obligación de pagar caras licencias. Además es sencillo de implementar y no requiere de mucho mantenimiento, razón por la cual es muy apreciado como base de datos de sitios web y para aplicaciones que buscan alta eficiencia con poco esfuerzo; sin embargo, no ofrece la misma robustez de sus competidores cuando lo que se busca es escalabilidad. Finalmente, SAP aparece también en esta categoría además de figurar en la de programación con su insigne lenguaje ABAP, ya que por ser un ERP, cuenta con su propio motor interno de bases de datos. Si bien SAP, al igual que DB2 y Sybase, tuvieron poca repetición en la muestra, las empresas que los usan son grandes, por lo cual se requiere una cantidad significativa de personal capacitado. 
Figura 8. Gestores de Bases de Datos más utilizados

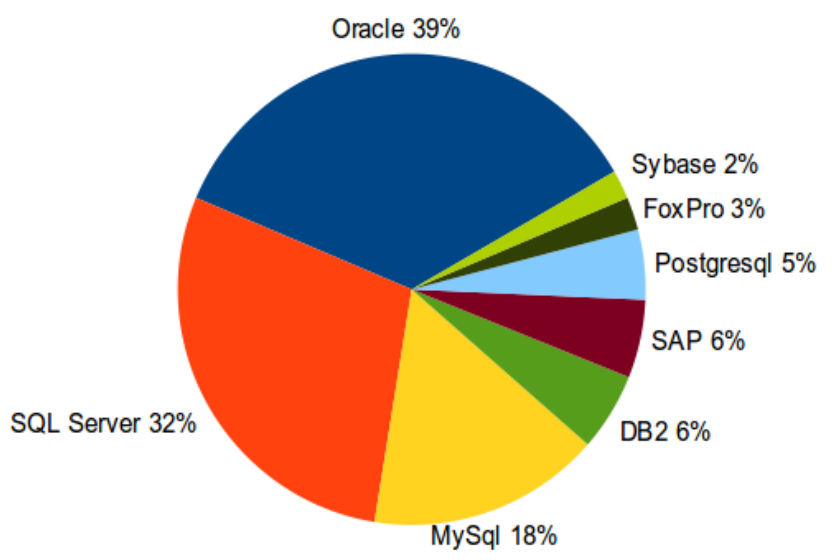

Fuente: elaboración propia.

\section{Metodologías de desarrollo}

Con respecto al dominio de metodologías de desarrollo de sistemas, se determinó que el mercado nacional pocas veces busca analistas que estén especializados solamente en dicha temática. En cambio, se espera que los mismos programadores las conozcan y sepan aplicarlas eficientemente en su trabajo. Las metodologías más comúnmente deseadas son:

- RUP: Proceso Unificado Racional.

- RAD: Desarrollo Rápido de Aplicaciones.

- AGILE: Desarrollo Ágil de aplicaciones.

- Extreme: Programación Extrema.

- ITIL: Biblioteca de Infraestructura de Tecnologías de Información.

- TOGAF: The Open Group Architecture Framework o Arquitectura del Marco de trabajo de "El Grupo Abierto".

- ETL: Extraer, Transformar y Cargar (los datos).

- PDM: Manejo de Datos de Productos.

- Diagramación apoyada por uml (Lenguaje Univer-sal de Modelado).

- Diagnóstico y diagramación de procesos de negocio.

\section{Servicios de reportería}

Todos los sistemas deben proveer al usuario de funciones necesarias para crear e imprimir reportes de información. Por tal razón, las empresas asumen que todos los programadores deben conocer algunas tecnologías para cumplir tales objetivos. De hecho, sólo un $15 \%$ de las empresas lo exige como un requisito explícito, aunque el $85 \%$ restante lo considera un valor agregado muy importante. Las tecnologías de reportería, ordenadas por importancia en el mercado local, son:

- Microsoft Excel (Mediante interoperatividad, para crear tablas dinámicas).

- Crystal Reports.

- Oracle Reports.

- Generador PDF.

- SQL Server Reporting.

- Jasper Reports.

- Report Builder.

\section{Servicios de pruebas}

La capacidad de un informático para crear pruebas de componentes de software se considera una característica "muy deseable"; no obstante, tal área se detectó como poco explorada en nuestro país. No más de un $3 \%$ de las empresas exigen explícitamente este tipo de tecnologías, entre las cuales se puede mencionar:

- Capibara

- Unit Testing

- Regression Test

- Rational ClearQuest

\section{Redes informáticas}

En el área de redes informáticas, las certificaciones Cisco Certified Network Associate - CCNA y Cisco Certified Network Professional-ccnp de Cisco son bien valoradas y suelen ser garantía de competencia. Las posiciones laborales de administrador de redes son pocas, debido a que uno o dos profesionales de este tipo bastan para dar soporte a la infraestructura de la mayoría de empresas medianas o grandes; ahora bien, como el personal especializado y debidamente certificado en tales áreas es relativamente escaso, existen buenas oportunidades de colocación. Aunado a estos conocimientos, se vuelve deseable que los profesionales dominen otras áreas de tecnología informática como:

- Configuración de servidores Apache, Apache Cordoba, Glassfish, Tomcat e iss.

- Configuración de cms como Drupal y Joomla.

- Hadoop con Apache y HortonWorks.

- Conocimiento sobre calidad de servicios "QoS".

- Aceleradores hтTP como Varnish.

- Herramientas de monitoreo y servicios. 
- Configuración de servicios de voz sobre IP.

\section{Modelo de niveles de competencias}

Según los perfiles informáticos que las 304 empresas de la muestra manifestaron tener, se elaboró una matriz de competencias profesionales deseables, como paso concluyente de la etapa de diagnóstico:

Tabla 1. Matriz de competencia de profesionales deseables según los perfiles profesionales requeridos por las empresas de El Salvador

\begin{tabular}{|c|c|c|c|c|c|}
\hline \multirow[b]{2}{*}{ ÁREAS DE CONOCIMIENTO } & \multicolumn{5}{|c|}{ PERFIL DEL PROFESIONAL DE LA INFORMÁTICA } \\
\hline & Programador & $\begin{array}{l}\text { Analista de } \\
\text { sistemas }\end{array}$ & $\begin{array}{l}\text { Administrador de } \\
\text { Bases de Datos }\end{array}$ & $\begin{array}{l}\text { Administrador } \\
\text { de Sistemas (it) }\end{array}$ & $\begin{array}{l}\text { Administrador } \\
\text { de redes }\end{array}$ \\
\hline Programación & Avanzado & $\begin{array}{l}\text { Intermedio- } \\
\text { avanzado }\end{array}$ & Intermedio & Intermedio & Básico \\
\hline Bases de datos & Intermedio-avanzado & Intermedio & Avanzado & Intermedio & Básico \\
\hline $\begin{array}{l}\text { Sistemas Operativos de } \\
\text { servidores }\end{array}$ & Básico & Básico & Intermedio & Avanzado & $\begin{array}{l}\text { Intermedio- } \\
\text { avanzado }\end{array}$ \\
\hline $\begin{array}{l}\text { Metodologías de } \\
\text { desarrollo de sistemas }\end{array}$ & Intermedio-avanzado & Avanzado & Básico & Básico & Básico \\
\hline $\begin{array}{l}\text { Herramientas para } \\
\text { desarrollo Web } 2.0\end{array}$ & Intermedio- avanzado & Intermedio & Básico & Básico & Básico \\
\hline Configuración de redes & Básico & Básico & Básico & $\begin{array}{l}\text { Intermedio- } \\
\text { avanzado }\end{array}$ & Avanzado \\
\hline $\begin{array}{l}\text { Conocimiento sobre } \\
\text { hardware }\end{array}$ & Básico & Básico & Intermedio & Avanzado & Avanzado \\
\hline Desarrollo para móviles & Intermedio-avanzado & Intermedio & Intermedio & Básico & Básico \\
\hline
\end{tabular}

Fuente: elaboración propia.

Como es de esperar, ningún profesional sería capaz de dominar a nivel avanzado cada una de estas áreas; no obstante, es necesario que los estudiantes de ingenierías y licenciaturas relativas a los sistemas informáticos, se especialicen en al menos una de ellas y tengan conocimientos intermedios en otras que le sean complementarias.

El perfil de "mantenimiento de hardware" se descartó, debido a que las empresas buscan más que todo a técnicos en esa área para cubrir las plazas de soporte. Así como también se determinó que no es requerido que los ingenieros en sistemas estén capacitados en herramientas de computadora para el diseño gráfico, ya que actualmente existen licenciaturas especializadas en ello.

Fruto del diagnóstico realizado durante esta investigación, se elaboró un modelo de competencias que 
cumpla con los requerimientos actuales del mercado informático salvadoreño, cuyo temario ser resume a continuación:

\section{Competencias sobre lenguajes de programación}

1. Instalación de frameworks y librerías de plataforma.

2. Conexiones a múltiples proveedores de bases de datos.

3. Control integrado de transacciones y concurrencia.

4. Manejo de estructuras de datos orientadas a objeto.

5. Ejecución de instrucciones SQL y procedimientos almacenados.

6. Creación de interfaces adaptativas para los usuarios.

7. Programación orientada a objeto.

8. Desarrollo de aplicaciones multicapa para separar la lógica de negocios y la arquitectura de acceso a datos del modelo de vista del usuario.

9. Desarrollo de modelos de entidades.

10. Manejo eficiente de big data.

11. Implementación de subsistemas para pruebas de funcionamiento de modelos de objetos.

12. Desarrollo de aplicaciones Web que reutilicen el modelo de clases de negocios.

13. Creación y utilización de WebServices basados en protocolo SOAP.

14. Manejo de seguridad, control de sesiones y auditorías de datos.

15. Desarrollo de módulos de reportería con soporte a múltiples formatos.

16. Integración de frameworks para la persistencia de datos y desarrollo ágil:
a. Spring (Java y .Net).
b. Hibernate (Java y .Net).
c. ASP.Net MVC.
d. DevExpress (.Net).
e. LinQ (.Net).
f. Node.js (Javascript).
g. Struts (Java).
H. JPA (Java).

17. Aplicación de herramientas colaborativas para compartir código fuente y controlar versiones.

18. Habilidad de investigación e implementación de nuevas tecnologías.

19. Desarrollo a partir de modelos conceptuales y no por codificación directa.

20. Buenas prácticas de codificación por indentado, sumarizado, comentado y documentado.
21. Comprensión y reutilización de código preexistente.

22. Conocer más de un lenguaje de programación, al menos dos en nivel Intermedio-Avanzado.

II. Competencias sobre gestores de bases de datos

1. Instalar y configurar el software servidor de bases de datos.

2. Configuración y sincronización de servicios en memoria y en la nube.

3. Seguridad:

a. Creación de roles.

b. Administración de usuarios.

c. Control de conexiones.

d. Otorgar permisos sobre objetos.

e. Uso de herramientas para el manejo de la seguridad de la información.

f. Realización de auditorías de datos.

4. Conocimientos de instrucciones para definición y manipulación de datos:
a. Consultas.
b. Sub consultas.
c. Tablas.
d. Objetos del sistema.
e. Clusters.
f. Índices.
g. Vistas.
h. Secuencias.
i. Paquetes.
j. Procedimientos almacenados.
k. Funciones.
l. Control de transacciones.

5. Conocimientos Intermedios-Avanzados de lenguajes procedurales como PL/SQL Y TRANSACT-SQL.

6. Creación de Servicios OLAP.

7. Manejo eficiente de big data.

8. Configuración de clusters, RAID y Data Guards.

9. Configuración de copias de respaldo automáticas a diversos medios y restauración de datos.

10. Aplicación de reglas de normalización y optimización SQL.

11. Aplicación de metodologías para el diseño de bases de datos eficientes que cumplan con los estándares de la industria.

12. Conocimientos sobre migraciones de bases de datos.

13. Habilidad para aplicar reglas de negocio a restricciones de tablas, procedimientos almacenados y funciones.

14. Uso de patrones de diseño.

15. Uso de expresiones regulares para validación de 
datos.

16. Implementación de servicios de reportería para consulta local y vía email.

17. Creación de servicios de formularios.

18. Configuración de Collations.

19. Conocimientos sobre la arquitectura de ERP's.

20. Implementación de ERP's para empresas.

21. Programación para ERP's.

\section{Competencias sobre herramientas para desarrollo web 2.0}

1. Codificación de HTmL5.

2. Codificación de Javascript.

3. Codificación de Jquery.

4. Codificación de Jquery Mobile.

5. Diseño de estilos con css.

6. Codificación de AJAX.

7. Codificación de XML

8. Codificación de Json.

9. Implementación de framework PrimeFaces para Java.

10. Implementación de framework para PHP:
a. Codelgniter.
b. Bootstrap.
c. Laravel.
d. Angular.
e. Symfony.
f. Zend.

11. Integración de Google Apps a la empresa.

12. Interacción con API Facebook.

13. Interacción con API Twitter.

14. Interacción con Wordpress.

15. Configuración de Microsoft Sharepoint.

16. Configuración de Apache.

17. Configuración de Apache Cordova.

18. Configuración de GlassFish.

19. Configuración de Івм WebSphere.

20. Configuración de IIs.

21. Configuración de Tomcat.

\section{Competencias sobre metodologías de desarrollo} de sistemas

1. Conocimiento sobre conceptos de inteligencia de negocios.

2. Creación de diagramas de procesos para el diagnóstico de reglas de negocio.

3. Aplicación de reglas de negocio sobre Software.

4. Diseño de sistemas multicapa.

5. Creación de diagramas Entidad-Relación.

6. Describir mediante diagramación, la arquitectura de hardware de un sistema.
7. Describir mediante diagramación, la arquitectura de software de un sistema.

8. Diagramación con Lenguaje Universal de Modelado -UML

a. Diagramas de estructura:
a. Diagrama de clases.
b. Diagrama de objetos.
c. Diagrama de componentes.
d. Diagrama de estructura compuesta.
e. Diagrama de paquetes.
f. Diagrama de implementación.

b. Diagramas de comportamiento:
a. Diagrama de casos de uso.
b. Diagrama de actividad.
c. Diagrama de estados de máquina.

c. Diagramas de interacción:

a. Diagrama de secuencias.

b. Diagrama de comunicaciones.

c. Diagramas de tiempo.

d. Diagramas de interacción general.

9. Uso de herramientas de Diseño Asistido por Computador-CASE.

10. Conocimiento sobre el modelo clásico de desarrollo de sistemas.

11. Implementación de RUP (Proceso Unificado Racional).

12. Implementación de RAD (Desarrollo Rápido de Aplicaciones).

13. Implementación de Extreme (Programación Extrema).

14. Implementación de ITIL (Biblioteca de Infraestructura de Tecnologías de Información).

15. Implementación de tOGAF (Esquema de Arquitectura del Open Group).

16. Implementación de ETL (Extracción, Transformación y Carga).

17. Implementación de PDM (Gestión de Datos de Producto).

18. Implementación de AGILE (Desarrollo Ágil).

\section{Competencias sobre sistemas operativos para servidores}

1. Instalación y configuración del servidor.

2. Administración local y remota.

3. Administración de sistemas de archivos.

4. Configuración de redes y dominios.
a. IPv4.
b. IPv6.

5. Configuración de seguridad.

6. Virtualización: 

a. VMWare.
b. Citrix XenServer.
c. Oracle VirtualBox.
d. Microsoft Hyper-V.

7. Ejecución de copias de seguridad y restauraciones.

8. Configuraciones de servicios basados en Lightweight Directory Acces Protocol -LDAP.

9. Configuración de servicios Web:
a. Servidor de páginas/aplicaciones Web.
b. Correo.
c. FTP (File Transfer Protocol).
d. Firewall.
E. DNS (Domain Name Service).
F. DHCP (Dinamic Host Configuration Protocol).
g. Proxy.
h. File Server.
i. Stream Server.
J. VPN (Virtual Private Network)
k. Monitoring.

10. Configuración de clusters.

11. Configurar cifrado y Auditoría Avanzada.

12. Planeación de la capacidad y ajustes de rendimiento.

13. Migración de datos entre versiones.

14. Gestionar procesos en memoria.

15. Administración de cuotas.

16. Aplicación de actualizaciones.

17. Creación de scripts de consola.

18. Administración de servicios de impresión.

19. Aplicar hacking ético a redes y servicios

\section{Competencias sobre redes informáticas}

1. Creación de redes de datos con IP.

2. Configuración de Switches para LAN (Local Area NetWork).

3. Creación de redes VLAN.

4. Administración de direcciones IP para IPV4 e IPV6.

5. Conocimiento sobre protocolos de ruteo.

6. Configuración de routers para redes IP.

7. Administración de Servicios IP.

8. Redirección de paquetes con NAT (Network Address Translation).

9. Aplicación de técnicas de seguridad.

10. Conocimiento sobre arquitectura de defensa de amenazas.

11. Detección y corrección de problemas en redes informáticas.

12. Configuración de redes waN (Wide Area Network).

13. Aseguramiento de accesos.
14. Configuración de redes VPN.

15. Diseño y aplicación de reglas de Firewall.

16. Configuración de IPS.

17. Manejo y monitoreo de sistemas en la nube.

18. Implementación de Mobile Networks.

\section{CONCLUSIONES}

En la actualidad, es urgente orientar la educación a un modelo realmente basado en competencias. La cultura enciclopedista de memorización y repetición no faculta a ningún estudiante para afrontar los retos de esta era de las sociedades de la información. En cambio, un sólido aprendizaje basado en competencias, permitiría al profesional salvadoreño desenvolverse en forma efectiva con base a tres dimensiones fundamentales: la cognoscitiva, la procedimental y la actitudinal.

El aspecto cognoscitivo tiene que ver con "aprender a aprender", es decir, adquirir la capacidad para construir constantemente nuevos aprendizajes, dadas las rápidas variaciones presentes en la industria de la informática. Además, las empresas esperan que los profesionales de sistemas sean verdaderos autodidactas, bastándose a sí mismos para implementar nuevas tecnologías y soluciones innovadoras. Hoy en día no basta el simple saber, sino que es imprescindible trascender los límites de la educación universitaria hacia un perfeccionamiento permanente.

Por su parte, la dimensión procedimental de las competencias se conforma por la correcta aplicación práctica de los conocimientos. Las empresas esperan que los informáticos sean capaces de programar diversas aplicaciones, configurar servicios, instalar sistemas operativos, entre otras actividades que resuelvan problemas complejos de la realidad. Esto quiere decir que el profesional debe "saber hacer" cosas concretas, con eficiencia y eficacia.

Por último, las empresas ya no se conforman sólo con el saber y el hacer. Ahora demandan que los profesionales tengan buena actitud para aprender lo que sea necesario, que asuman retos sin pretextos, que tengan valores, que sean honestos, diligentes, serviciales y que además "sepan ser" buenas personas para favorecer el clima organizacional y el trabajo en equipo.

\section{RECOMENDACIONES}

A los estudiantes: Tomar muy en cuenta los resultados del presente estudio para trazarse metas claras a alcanzar 
en cuanto a conocimientos y habilidades técnicas, siendo consecuentes con éstas mismas a través del esfuerzo constante y un real interés por el aprendizaje a fondo de las nuevas tecnologías. Aunado a ello, es necesario comprender que con el trabajo cotidiano y de calidad), se honra a Dios y se beneficia a la sociedad.

A los docentes: Orientar a sus alumnos hacia la obtención integral de competencias que sean valiosas en el mundo laboral actual según tres dimensiones fundamentales: cognoscitivas, operacionales y actitudinales. Para alcanzar este ideal será necesario desterrar las metodologías enciclopedistas y educar a los jóvenes para que sean autores de su propio desarrollo, utilizando el modelo de competencias propuesto por este estudio como un indicador de logro en sus respectivas materias.

A la universidad: Revisar constantemente el plan de la carrera de Ingeniería en Sistemas Informáticos y sus asignaturas, así como fomentar el trabajo en equipo entre docentes para encontrar estrategias que permitan implementar un verdadero modelo de formación profesional basado en competencias. Además, se recomienda incursionar en la creación de cursos y pruebas para la certificación de competencias de los estudiantes.

\section{REFERENCIAS}

Bernal, C. (2006). Metodología de la Investigación. México: Pearson Education.

Casanovas, J., y Colom, J. (2002). El Libro Blanco de la Ingeniería en Informática: el proyecto EICE. España: Agencia Nacional de Evaluación

CEPAL y OIT. (2015). Coyuntura laboral en América Latina y el Caribe. Comisión Económica para América Latina y el Caribe, onu. Recuperado de http://repositorio.cepal.org/bitstream/handle/11362/38051/S1500311_es.pdf?sequence=1

Corominas, E. (2006). Percepciones del profesorado ante la incorporación de las competencias ge- néricas en la formación universitaria. Revista de Educación, 341, 301-336. Recuperado de: http://dugldoc.udg.edu:8080/bitstream/handle/10256/9861/PercepcionesProfesorado.pdf

Giraldo, G. (2009). Incorporación de las competencias al modelo de construcción de currículos de ingeniería basados en problemas: el caso de la ingeniería de sistemas. Recuperado de: http://www.lsi.upc.edu/ balqui/compet.pdf

Juan A., J., Nieto, C., Suárez, M., Pérez, J., Cernuda, A., Luengo, C., Martínez, A., Riesco, M., Lanvín, F., Labra, J., Fondón, M., y Redondo, J. (2006). Definición de competencias específicas y genéricas del Ingeniero en Informática. Recuperado de http://listas.exa.unne.edu.ar/ matematica/metodos/8-sitios-material-interes/ Competencias\%20del\%20ing\%20Informatico.pdf

Pavez, A. (2000). Modelo de implantación de Gestión del Conocimiento y Tecnologías de Información para la Generación de Ventajas Competitivas. Recuperado de http://cdim.esap.edu.co/BancoMedios/Documentos\%20PDF/modelo\%20 de\%20implantaci\%C3\%B3n\%20de\%20gc\%20 y\%20ti\%20para\%20la\%20generaci\%C3\%B3n\%20 de\%20ventajas\%20competitivas.pdf

Pérez, R., y Catalán, R. (2004). El modelo pedagógico de la formación universitaria en el crédito europeo: una innovación en la materia "Informática aplicada a la investigación educativa. Recuperado de http:// dehesa.unex.es:8080/xmlui/bitstream/handle/10662/1732/1695-288X_3_2_81.pdf?sequence $=1$

Revista CEPAL. (2005). Competencias técnicas de los trabajadores informáticos. Comisión Económica para América Latina y el Caribe, onU. Recuperado de http://repositorio.cepal.org/bitstream/ handle/11362/37474/RVE87_es.pdf?sequence=1

Ricoy, M., Feliz, T., y Sevillano, M. (2010). Competencias para la utilización de las herramientas digitales en la sociedad de la información. Recuperado de http://revistas.uned.es/index. php/educacionxx1/article/view/283/239

Sánchez, F., Sancho M. R., Botella, P., García, J., y Aluja, T. (2008). Competencias profesionales del Grado en Ingeniería Informática. Recuperado de http://www.lsi.upc.edu/ balqui/ 
compet.pdf

Schmal, R., y Ruiz, A. (2008). Una metodología para el diseño de un currículo orientado a las competencias. Revista chilena de ingeniería, vol. 16,(1), 147-158. Recuperado de http://www. scielo.cl/pdf/ingeniare/v16n1/ART04.pdf

Simón, R., y Ruiz, A. (2010). Un modelo para la gestión de una escuela universitaria orientada a la formación basada en competencias. Recuperado de http://www.scielo.org.co/pdf/ cadm/ v22n39/v22n39a13.pdf

Urquiza, F. (2007). Aplicación de modelos de competencias a la gestión de sistemas de información. Recuperado de http://www.ati.es/IMG/ pdf/UrquizaVol3Num1.pdf
Villa, A. (2007). Aprendizaje basado en competencias: una propuesta para la evaluación de las competencias genéricas. Bilbao, España: Ediciones Mensajero.

Yoguel, G., Novik, M., Milesi, D., y Roitter, S. (2004). Información y conocimiento: la difusión de TICS en la industria manufacturera argentina. Recuperado de http://www.littec.ungs.edu. ar/SSI2004/7_Yoguel\%20et\%20al.pdf

Zalba, E., Gutiérrez, N., y Deamici, C. (2010). Educación Basada en Competencias: Desarrollos conceptuales y experiencias. Mendoza: Editorial Ediunc. 